\title{
ACCIONES DE PREVENCIÓN Y CONTROL DE LA LEGIONELOSIS: UN RETO PARA LA SALUD PÚBLICA ESPAÑOLA
}

\author{
Enrique Gea-Izquierdo1,a, Edward Mezones-Holguín²,3,b, Luis Haro-García 4,c
}

\begin{abstract}
RESUMEN
La legionelosis es una enfermedad respiratoria con origen en sistemas que formen aerosol y que contenga el agente biológico Legionella sp. En las últimas décadas se ha desarrollado en España un marco normativo para su prevención y control. El presente artículo expone la epidemiología de la legionelosis y la importancia del control de la transmisión de la bacteria en la lucha contra la enfermedad. Para ello, se hace patente la revisión de las instalaciones críticas y la inclusión de otras nuevas en la legislación preventiva así como la estimación del riesgo, la mejora en los procesos de diagnóstico y el avance en nuevos protocolos de prevención.
\end{abstract}

Palabras clave: Legionelosis; Legionella; Legislación; España; Salud Pública (fuente: DeCS BIREME).

\section{ACTIONS FOR LEGIONELLOSIS PREVENTION AND CONTROL: A CHALLENGE FOR PUBLIC HEALTH IN SPAIN}

\begin{abstract}
Legionellosis is a respiratory disease originating in systems that produce aerosol and contain Legionella $s p$. In recent decades, Spain has developed a regulatory framework for prevention and control of legionellosis. This article describes the epidemiology of legionellosis and the importance of controlling the transmission of bacteria in the fight against the disease. In that regard, it becomes clear the role of reviewing critical facilities and the inclusion of new ones in the preventive legislation, the estimation of risk, and the improvement in the diagnostic processes and progress in new prevention protocols.
\end{abstract}

Key words: Legionellosis; Legionella; Legislation; Spain; Public Health (source: MeSH NLM).

\section{INTRODUCCIÓN}

La legionelosis es una enfermedad respiratoria originada por la bacteria Legionella sp. de la familia Legionellaceae. Este microorganismo tiene forma de bacilo y dimensiones que oscilan entre 1,5 - $5 \mu \mathrm{m}$ de largo por 0,5- 0,7 $\mu \mathrm{m}$ de ancho, con uno o más flagelos polares o subpolares. Más de la mitad de las especies de Legionella sp. han estado implicadas en infección humana(1); y la Legionella pneumophila es causante de aproximadamente $85 \%$ de las infecciones (2). La familia comprende un género, Legionella, con 40 especies y más de 50 serogrupos; mientras que para la especie Legionella pneumophila se han descrito 14 serogrupos ${ }^{(3)}$. En la legionelosis existe un periodo desde la infección hasta la aparición de los primeros síntomas, que suele ser de 2-10 días (5 a 6 días es más frecuente); aunque en algunos casos es variable, pudiéndose describir periodos de hasta tres semanas con una mortalidad de $10-15 \%$. La Legionella sp. es una bacteria ampliamente distribuida en los ambientes acuáticos tanto naturales como antrópicos; tolerando diversas condiciones físicoquímicas. Existe alguna especie como Legionella longbeacheae que ha sido aislada en tierra ${ }^{(4)}$, aunque de manera ocasional, siendo además una especie que se presenta fuera de Europa.

Cuando la bacteria coloniza los medios no naturales es capaz de desarrollarse siempre que se determinen elementos amplificadores, mostrando riesgo elevado si hay producción de aerosoles que provoquen su dispersión ${ }^{(5)}$. Sin embargo, el hecho de que se identifique la bacteria en un sistema o dispositivo determinado no es condicional que se desarrolle la enfermedad. El microorganismo tiene que alcanzar determinado nivel

\footnotetext{
Cátedra de Seguridad y Salud en el Trabajo, Universidad de Málaga. Málaga, España.

Unidad de Análisis y Generación de Evidencias en Salud Pública, Centro Nacional de Salud Pública, Instituto Nacional de Salud. Lima, Perú.

Escuela de Medicina, Universidad Peruana de Ciencias Aplicadas. Lima, Perú.

Departamento de Salud Pública, Facultad de Medicina, Universidad Nacional Autónoma de México. México DF, México.

Biólogo doctor en Ciencias Biológicas, máster en Medicina Preventiva y Salud Pública, máster en Seguridad y Salud en el Trabajo; ${ }^{\text {b }}$ médico maestro en Ciencias en Epidemiología Clínica; ${ }^{c}$ médico especialista en Medicina Familiar y Salud en el Trabajo, doctor en Ciencias
} 
de concentración para la posible afectación de las personas existiendo, además, variabilidad en el tiempo de exposición.

La infección por Legionella sp. puede adquirirse fundamentalmente en dos grandes ámbitos: el comunitario y el hospitalario. En ambos casos la enfermedad puede estar asociada con varios tipos de instalaciones y edificios; presentarse en forma de brotes o casos agrupados asi como en casos esporádicos o aislados. De hecho, la frecuencia de aparición de la enfermedad es bastante relevante y constituye uno de los tipos de neumonía infecciosa más frecuente en el mundo tecnológicamente desarrollado. El control y la prevención de desarrollo de la enfermedad se fundamentan en la aplicación de medidas preventivas en las fuentes de origen y en el mantenimiento preventivo de los diferentes sistemas.

Si bien la presentación habitual de la enfermedad es en forma de casos esporádicos, cada vez es más frecuente la aparición en forma de casos agrupados o brotes con un gran número de afectados. El origen de un gran número de brotes comunitarios con un elevado número de casos suelen estar asociados a las torres de refrigeración; así también, la enfermedad es habitual en pacientes hospitalizados. Sin embargo, el reconocimiento de brotes de legionelosis es algo inusual, cuando ocurren se muestran oportunidades para comprender la epidemiología de la enfermedad e impulsar mejoras en las estrategias de su prevención. Debido al aumento en la movilidad internacional, se hace difícil realizar investigaciones epidemiológicas para la localización y eliminación de las fuentes de infección. Es objeto de debate que las personas deberían estar informadas sobre las distintas fuentes ${ }^{(6)}$. Especialmente notable es la notificación de casos conocidos como "legionelosis del viajero" que, en países como España, puede tener repercusión importante sobre el desarrollo del turismo. La Legionella sp. causa $0,5-5 \%$ de los casos de neumonía adquirida en la comunidad, donde 15\% de estos requieren hospitalización ${ }^{(7)}$. Otros autores ${ }^{(8)}$ plantean que la incidencia de la infección por Legionella es altamente variable, constituyendo entre el 1 y $27 \%$ de las neumonías adquiridas en la comunidad.

\section{EPIDEMIOLOGÍA DE LA LEGIONELOSIS}

La enfermedad muestra una distribución mundial con representación en América del Norte, Suramérica, Asia, África, Australia y Europa ${ }^{(9-14)}$. A finales de los años noventa, en Europa y según la Organización Mundial de la Salud ${ }^{(15)}$, se advirtió un aumento en el número de países con aportación de datos referida a la enfermedad, aun siendo muy variable su incidencia. Las tasas correspondientes al año 1999 variaban desde 19,5 por millón de habitantes en Bélgica a uno por millón de habitantes en Portugal; mientras que España presentaba una tasa de 7,8. Sin embargo, estos datos podrían estar infravalorados con respecto a la incidencia real, debido a la existencia de Legionella sp. no consideradas por estar desvinculadas del sector turístico. En Europa, las cifras de incidencia notificada varían mucho de un país a otro, dependiendo de las características de los sistemas de vigilancia. El mayor número de casos es de categoría desconocida (importante objeto de estudio), aconteciendo el ámbito comunitario en segundo lugar. Convendría resaltar la importancia de la legionelosis relacionada con "los viajes al extranjero" y, al contrario de como se podría pensar, el ámbito intrahospitalario presenta un nivel inferior.

La legionelosis es una enfermedad de declaración obligatoria en el estado español. El Real Decreto 2210/1995 (16), por el que se creó la Red Nacional de Vigilancia Epidemiológica, establece la declaración semanalparaestaenfermedad,condatosepidemiológicos básicos. Antes de 1996 no se consideraba enfermedad de declaración obligatoria a nivel nacional. Desde 1997, año de introducción de la legionelosis en la lista de enfermedades de declaración obligatoria, la incidencia de la enfermedad ha presentado una tendencia creciente hasta el año 2002, con una tasa de crecimiento anual en ese período del $52,5 \%$. Este aumento en el número de casos declarados está relacionado con la amplia difusión del uso de la prueba de búsqueda de antígenos en orina como técnica diagnóstica (en nuestro país se aceptó como diagnóstico de caso confirmado en 1999) y la progresiva sensibilización en toda la comunidad científica por un mejor diagnóstico, control y prevención de la enfermedad. El año 2000 la tasa de incidencia fue de 1,9 por 100000 habitantes que, frente al año 1999, supuso un incremento del $74 \%$. A partir del año 2002 hasta el año 2009, se presenta una tendencia conservadora de las tasas de incidencia de la legionelosis.

\section{IMPORTANCIA DEL DESARROLLO LEGISLATIVO EN LA PREVENCIÓN DE LA LEGIONELOSIS}

A nivel mundial, Gran Bretaña es uno de los primeros países en implantar legislación y orientaciones técnicas para minimizar el riesgo de Legionella sp. (17) En España, debido a los brotes aparecidos en las distintas comunidades autónomas y a la alarma social causada (no siempre correspondida con la problemática sanitaria que se le quiere dar), se impone una revolución en cuanto al tratamiento legislativo de la prevención de la legionelosis. 
La Constitución Española ${ }^{(18)}$, en su artículo 43, reconoce el derecho a la protección de la salud y determina que compete a los poderes públicos organizar y tutelar la salud pública a través de medidas preventivas y de las prestaciones y servicios necesarios. El primer escalón de desarrollo preventivo lo constituye la Ley de Prevención de Riesgos Laborales ${ }^{(19)}$ y sus normas complementarias, como instrumento de protección de la seguridad y la salud. Esta Ley es cita ineludible y pilar direccional del desarrollo del marco normativo preventivo español, siendo igualmente de obligada mención el Reglamento de los Servicios de Prevención (20). En la evolución histórica legislativa, la referencia inicial comunitaria es la normativa publicada por la Comunidad de Madrid (21), por la que se regulan los criterios higiénico-sanitarios que deben reunir los aparatos de transferencia de masa de agua en corriente de aire, y aparatos de humectación para la prevención de la legionelosis.

Desde la óptica de la Salud Pública se consideran como elementos críticos ${ }^{(22)}$ las torres de refrigeración de agua, los condensadores evaporativos y otros equipos de intercambio aire-agua. Esto se debe a la capacidad de producir aerosoles, que pueden transportar la bacteria hacia el medioambiente cuando tales dispositivos se encuentran en malas condiciones. Si se repasan los brotes notificados en España durante los años 19892000 (años en que se produce el inicio del desarrollo de la normativa) se identifican 54 brotes, con 22 ocasiones donde el sistema de agua caliente sanitaria estuvo implicado. Curiosamente, las torres de refrigeración que son las que más alarma social causan, únicamente están relacionadas con 13 brotes de tipo comunitario abierto, considerándose, a veces, poco relevantes los ligados a otros sistemas. La normativa nacional de prevención y control de la legionelosis (23) describe el ámbito de aplicación, considerando las instalaciones, y su probabilidad de proliferación y dispersión de Legionella sp.

\section{DESARROLLO DE LA EVALUACIÓN DE RIESGOS, ESTIMACIÓN DEL RIESGO, CRITERIOS DE DIAGNÓSTICO Y NUEVOS PROTOCOLOS}

La evaluación y el control de la Legionella sp. es tema en constante renovación que en algunos países adopta gran importancia, donde quizás exista cierta relación entre el desarrollo de la enfermedad y el nivel de industrialización. Existen otros en los que, o no se conocen casos, o no están descritos, mientras que en alguno es un hecho infrecuente (24). En concreto, la vigilancia continuada; la definición de estrategias, y su puesta en práctica; pueden mostrar resultados positivos para la prevención de la legionelosis ${ }^{(25)}$. Es cierto que las condiciones de mantenimiento de los equipos así como las condiciones atmosféricas reinantes pueden tener un papel relevante; pero, en el medioambiente urbano la enfermedad se asocia usualmente con diseños deficientes en los edificios; construcción inapropiada, y negligencia en el control de la calidad del agua de las instalaciones.

En la estimación del riesgo, uno de los componentes prioritarios de la investigación epidemiológica es la identificación de la asociación causal entre determinado factor de riesgo, y daño para la salud, encontrando dificultades para la asignación del grado de exposición. Adicionalmente, los efectos sobre la salud pueden ser diferentes si afecta a edades tempranas o tardías, al estado inmunológico de la persona, si se presentan picos en la exposición y otros. Igualmente, el tipo de sistema influye en el riesgo por Legionella sp. (Tabla 1) variando de unos dispositivos a otros.

El criterio de diagnóstico de laboratorio(26) es aquel compatible con la definición clínica de caso;y cualquiera de los diagnósticos microbiológicos considerados de confirmación como el aislamiento de cualquier especie o serogrupo de Legionella a partir de secreciones respiratorias, tejido pulmonar o sangre; seroconversión (aumento del título de anticuerpos en cuatro veces o más) con un segundo título mínimo de 128 frente a Legionella pneumophila serogrupo 1 por inmunofluorescencia indirecta, en sueros tomados en la fase aguda y convaleciente de la enfermedad; y detección de antígeno Legionella pneumophila serogrupo 1, en orina por RIA o ELISA. Las pruebas anteriores se complementan unas a otras, por lo que su realización aumenta la probabilidad de confirmar el diagnóstico, teniendo en cuenta que ninguna de las pruebas posee una sensibilidad del $100 \%$.

La incapacidad para detectar el patógeno con cultivos rutinarios y técnicas de tinción presenta un problema en curso, ya que se debe considerar la implementación de protocolos de cultivos especiales, y procedimientos de antígenos urinarios. El reconocimiento temprano de la bacteria conducirá a la iniciación de terapias antimicrobianas efectivas ${ }^{(27)}$. Igualmente, se plantea la necesidad de desarrollo de métodos preventivos que incidan en la producción basados en técnicas de inspección rápida y que ayuden al mantenimiento de los diferentes sistemas. Se considera que la forma más adecuada para garantizar la uniformidad de las actuaciones contempladas en los programas de vigilancia y control es la elaboración de un documento base para que los responsables de las instalaciones desarrollen su propio plan de autocontrol. 
Tabla 1. Definición de los sistemas/dispositivos en función del riesgo de Legionella sp.

\begin{tabular}{|c|c|}
\hline Alto riesgo & Bajo riesgo \\
\hline $\begin{array}{l}\text { Torres de refrigeración, condensadores } \\
\text { evaporativos y sistemas adiabáticos. }\end{array}$ & Agua fría sanitaria. \\
\hline $\begin{array}{l}\text { Agua caliente sanitaria. } \\
\text { Agua en recirculación y agitación permanente (con posible } \\
\text { inyección de aire): piscinas de recreo, spas, ciertos acuarios, } \\
\text { etc. }\end{array}$ & $\begin{array}{l}\text { Sistemas de agua contra incendios. } \\
\text { Equipos de humectación (incluso cavas de puros) y aerosolización } \\
\text { (inclusive refrigeración). Sistemas de enfriamiento evaporativo } \\
\text { domésticos. }\end{array}$ \\
\hline $\begin{array}{l}\text { Sistemas de lavado de automóviles mediante pulverización: con } \\
\text { lanzadera o similar. Túneles de autolavado. }\end{array}$ & $\begin{array}{l}\text { Fuentes ornamentales. } \\
\text { Riego por aspersión. }\end{array}$ \\
\hline $\begin{array}{l}\text { Instalaciones termales. } \\
\text { Equipos de terapia respiratoria. } \\
\text { Recintos de concurrencia pública, climatizados y sometidos a } \\
\text { aerosoles (estaciones, parques, etc.) }\end{array}$ & $\begin{array}{l}\text { Sistemas físicos de conducción de aire "tratado". } \\
\text { Camiones cisterna para riego de vías públicas. } \\
\text { Sistemas de distribución, bombas de agua y otros dispositivos } \\
\text { (p.ej. barcos que requieran reparación). }\end{array}$ \\
\hline $\begin{array}{l}\text { Locales, terrazas o similar es que usan agua en aerosol para } \\
\text { "enfriar" el ambiente (p.ej. toldos y ventiladores con aerosoles). } \\
\text { Procesos humidificadores usados en industria } \\
\text { (p.ej. pescaderías). }\end{array}$ & $\begin{array}{l}\text { Circuitos de aire acondicionado con pérdidas de condensado } \\
\text { (p.ej. coches). } \\
\text { Bandejas de condensado (p.ej. baterías de unidades de } \\
\text { tratamiento de aire y fan-coils). }\end{array}$ \\
\hline Sistemas con calentadores eléctricos y aerosoles de agua. & Unidades de dentistas. \\
\hline
\end{tabular}

\section{CONCLUSIONES}

El desarrollo normativo español para la prevención de la legionelosis ha sufrido una enorme evolución en las últimas décadas. Gracias a la instauración de medidas de mejora, control y protección mediante tratamientos de los distintos sistemas críticos, se ha avanzado en la prevención de la dispersión del agente biológico y los resultados de la enfermedad. La legislación aporta una reducción en la exposición a la Legionella, minimizando el posible riesgo mediante el control de las fuentes de origen. Sin embargo, atendiendo a su epidemiología, la enfermedad sigue presentándose como un reto que superar. La consecuencia de la conjunción de las medidas de salud pública y comunicación del riesgo, conducirán al mejor conocimiento y control de los mecanismos preventivos, remarcando la importancia del marco legislativo como medio frente a la aparición de la legionelosis. Al mismo tiempo, se requiere mayor inspección sobre las labores realizadas por parte de empresas especializadas autorizadas y las prestaciones que de ellas se desprenden, así como la cualificación de personal vinculado con la Administración. La existencia de novedosas instalaciones o sistemas como posibles fuentes de proliferación o dispersión de la bacteria, precisa que sean incluidas en el ámbito de aplicación del desarrollo de una nueva normativa española para la prevención y control de la legionelosis.

\section{REFERENCIAS BIBLIOGRÁFICAS}

1. Lo Presti F, Riffard S, Vandenesch F, Reyrolle M, Ronco $\mathbf{E}$, Ichai $\mathbf{P}$, et al. The first clinical isolate of Legionella parisiensis, from a liver transplant patient with pneumonia. J Clin Microbiol. 1997;35(7):1706-9.
2. Marston BJ, Lipman HB, Breiman RF. Surveillance for legionnaires' disease. Risk factors for morbidity and mortality. Arch Intern Med. 1994;154(21):2417-22.

3. Benson RF, Thacker WL, Wilkinson HW, Fallon RJ, Brenner DJ. Legionella pneumophila serogroup 14 isolated from patients with fatal pneumonia. J Clin Microbiol. 1988;26(2):382.

4. Travis TC, Brown EW, Peruski LF, Siludjai D, Jorakate P, Salika P, et al. Survey of Legionella species found in thai soil. Int J Microbiol. 2012;2012:218791. Epub 2012 Jan 12.

5. Gea-Izquierdo E. Cumplimiento de los protocolos de prevención de la legionelosis en edificios públicos. Rev Salud Publica (Bogota). 2009;11(1):100-9.

6. Kistler W, Fleisch F, Reinhart WH, Wieland T. [Legionellosis, a tourist problem?]. [Article in German]. Praxis (Bern 1994). 2002;91(31-32):1241-5.

7. Greig JE, Carnie JA, Tallis GF, Ryan NJ, Tan AG, Gordon IR, et al. An outbreak of legionnaires' disease at the Melbourne Aquarium, April 2000: investigation and case-control studies. Med J Aust. 2004;180(11):566-72.

8. Cosentini R, Tarsia P, Blasi F, Roma E, Allegra L. Community-acquired pneumonia: role of atypical organisms. Monaldi Arch Chest Dis. 2001;56(6):527-34.

9. Silk BJ, Moore MR, Bergtholdt M, Gorwitz RJ, Kozak NA, Tha MM, et al. Eight years of Legionnaires' disease transmission in travellers to a condominium complex in Las Vegas, Nevada. Epidemiol Infect. 2012;4:1-10.

10. Cabello H, Cortés C, Ruiz M, Jover E, Rivera F, Segovia E, et al. Neumonia adquirida en la comunidad. Comunicacion de 8 casos de neumonia grave por Legionella pneumophila serogrupo 1 en Chile. Rev Med Chil. 2002;130(3):309-13.

11. Lam MC, Ang LW, Tan AL, James L, Goh KT. Epidemiology and control of legionellosis, Singapore. Emerg Infect Dis. 2011;17(7):1209-15.

12. Mehiri-Zghal E, Essalah L, Ghariani A, Mahjoubi W, Reyrolle M, Meugnier $\mathbf{H}$, et al. [Molecular comparison of Legionella pneumophila serogroup 1 isolated in Tunisia]. [Article in French]. Pathol Biol (Paris). 2008;56(5):279-82. 
13. Brown J, Hort K, Bouwman R, Capon A, Bansal N, Goldthorpe I, et al. Investigation and control of a cluster of cases of Legionnaires disease in western Sydney. Commun Dis Intell. 2001;25(2):63-6.

14. Blatny JM, Fossum H, Ho J, Tutkun M, Skogan G, Andreassen $\mathbf{O}$, et al. Dispersion of Legionella-containing aerosols from a biological treatment plant, Norway. Front Biosci (Elite Ed). 2011;1(3):1300-9.

15. World Health Organization (WHO). Legionnaires' disease, Europe, 1999. Wkly Epidemiol Rec. 2000;75(43):347-52.

16. Real Decreto $2210 / 1995$, de 28 de diciembre, por el que se crea la Red Nacional de Vigilancia Epidemiológica. B.O.E. núm. 21 de 24 de enero de 1996.

17. Brundrett G. Controlling Legionnaire's disease. Indoor and Built Environment. 2003;12(1-2):19-23.

18. Constitución Española. B.O.E. núm. 311 de 29 de diciembre de 1978.

19. Ley $31 / 1995$, de 8 de noviembre, de Prevención de Riesgos Laborales. B.O.E. núm. 269 de 10 de noviembre de 1995.

20. Real Decreto 39/1997, de 17 de enero, por el que se aprueba el Reglamento de los Servicios de Prevención. B.O.E. núm. 27 de 31 de enero de 1997.

21. Orden 1187/ 1998, de 11 de junio, de la Consejería de Sanidad y Servicios Sociales de la Comunidad de Madrid, por la que se regulan los criterios higiénico-sanitarios que deben reunir los aparatos de transferencia de masa de agua en corriente de aire y aparatos de humectación para la prevención de la legionelosis. B.O.C.M. núm. 144 de 19 de junio de 1998.
22. Gea-Izquierdo E. Prevención de la legionelosis en sistemas de refrigeración de agua. Dyna. 2011;78(165):9-17.

23. Real Decreto $865 / 2003$, de 4 de julio, por el que se establecen los criterios higiénico- sanitarios para la prevención y control de la legionelosis. B.O.E. núm. 171 de 18 de julio de 2003.

24. Luna CM. Neumonía por Legionella, un hecho infrecuente en Argentina ¿Diferente epidemiología o marcador del subdesarrollo? [carta]. Medicina (B Aires). 1999;59(3):311-2.

25. Kelly AA, Danko LH, Kralovic SM, Simbartl LA, Roselle GA. Legionella in the veterans healthcare system: report of an eight-year survey. Epidemiol Infect. 2003;131(2):835-9.

26. Junta de Andalucía, Consejería de Salud. Protocolos de Alerta Epidemiológica. Sevilla: Dirección General de Salud Pública y Participación. Servicio de Vigilancia Epidemiológica y Evaluación. SVEA-Sistema de Vigilancia Epidemiológica de Andalucía; 2003.

27. Adams SL. Legionella: a new millennium bug. Clin Lab Sci. 1999;12(5):309-15.

Correspondencia: Enrique Gea-Izquierdo

Dirección: calle Doctor Ortiz Ramos s/n 29071. Málaga, España.

Teléfono: (34) 677454317

Correo electrónico: enriquegea@telefonica.net

\section{Consulte la versión electrónica de la} Revista Peruana de Medicina Experimental y Salud Pública en www.pubmed.gov

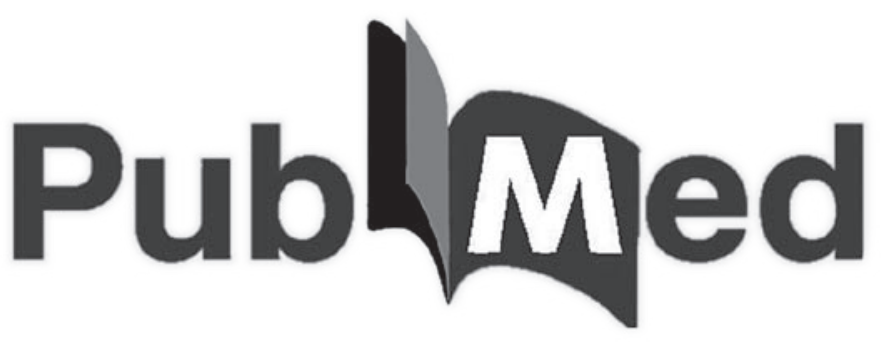

